\title{
PERFIL BIOSOCIODEMOGRÁFICO Y VALORACIÓN DE NECESIDADES EN PACIENTES EN DIÁLISIS PERITONEAL
}

\section{BIOSOCIODEMOGRAPHIC AND NEEDS ASSESSMENT PROFILE IN PATIENTS ON PERITONEAL DIALYSIS}

\author{
Jocelyn UlLoa VArela* \\ Magali RodRÍGUEZ VIDAL ${ }^{* *}$ \\ Mónica Cruz Pedreros ${ }^{* * *}$
}

\begin{abstract}
RESUMEN
Objetivo: El siguiente estudio pretende conocer las características biosociodemográficas y la satisfacción de necesidades de los pacientes en tratamiento con diálisis peritoneal del Hospital Las Higueras de Talcahuano, Chile. Material y método: Estudio cuantitativo, descriptivo, de corte transversal; la población de estudio estuvo constituida por 40 pacientes, se realizó una entrevista utilizando un instrumento que incluyó la evaluación de satisfacción de necesidades básicas, según Virginia Henderson, y factores biosociodemográfics. Resultados: El grupo de estudio estuvo compuesto en igual parte por hombres y mujeres, el promedio de edad correspondió a 48 años, más de la mitad vive sin pareja y es jefe de hogar, la mayoría tiene hijos, predominando los grupos familiares integrados por 4 o más personas $(57,5 \%)$. El mayor porcentaje de ellos ha cursado enseñanza media, el 22,5\% se encuentra trabajando y recibe remuneración. Casi la totalidad cuenta con saneamiento básico en sus domicilios y vive en sector urbano. Las principales necesidades insatisfechas corresponden a "eliminar bien" y "evitar peligros", las restantes se encuentran satisfechas entre el 57,5\% y 92,5\%; dentro de este rango las necesidades más afectadas fueron mantener la temperatura corporal y recrearse. Conclusión: Los resultados sugieren implementar y reforzar planes educativos más rigurosos a los ya existentes en nuestro centro asistencial, tendientes a evitar daños físicos y sicológicos, así como también mantener una buena eliminación corporal, a través de la retroalimentación en cada control o visita domiciliaria realizada al usuario a nuestro cuidado.
\end{abstract}

Palabras clave: Insuficiencia renal crónica, enfermedad renal, diálisis peritoneal, evaluación de necesidades, enfermería en nefrología.

\begin{abstract}
Objective: To know the biosociodemographic profile of patients on peritoneal dialysis Hospital Las Higueras in Talcahuano, Chile and the satisfaction of basic needs they have. Methods: Quantitative, descriptive and crosssectional desing. The sample consisted of 40 patients, an interview was conducted, using an instrument that included evaluation of basic needs, according to Virginia Henderson and biosociodemographic factors. Results: The study group was composed in equal parts by men and women, average age corresponded to 48 , more than half live without a partner and head of household, the majority have children, predominantly family groups consisting of 4 or more people (57.5\%). The highest percentage of them have completed high school, $22.5 \%$ are working and receives remuneration. Almost all have basic sanitation in their homes and live in urban areas.

\footnotetext{
* Enfermera. Unidad de Diálisis Hospital Las Higueras. Talcahuano, Chile. Email: joceulloa@gmail.com

** Enfermera. Docente Departamento de Enfermería. Universidad de Concepción, Concepción Chile. Email: magrodri@ udec.cl

${ }^{* * *}$ Enfermera. Docente Departamento de Enfermería Universidad de Concepción, Concepción Chile. Email: mocruz@udec.cl
} 
Major unsatisfied needs are "good elimination" and "avoid danger", the rest are satisfied between 57.5\% and $92.5 \%$, within this range needs were maintaining body temperature and recreation. Conclusion: Results suggest, implementing and enforcing more stringent educational plans than the existing ones in our medical center designed to prevent physical and psychological damage as well as maintain good body elimination, through feedback in each control or home visit to the user under our care.

Key words: Renal insufficiency chronic, renal disease, peritoneal dialysis, needs evaluation, nephrology nursing.

Fecha recepción: 05/11/12 Fecha aceptación: 31/01/14

\section{INTRODUCCIÓN}

La enfermedad renal crónica (ERC) constituye un importante problema de salud pública, cuya manifestación más avanzada es la enfermedad renal crónica terminal (ERCT), que requiere tratamiento sustitutivo de la función renal mediante diálisis, o trasplante.

Según estudios españoles al 2005, las causas más frecuentes de ERC correspondían a la Diabetes Mellitus (DM) e Hipertensión Arterial (HTA), que se han triplicado durante la última década (1), ya para el 2010, de acuerdo a informes publicados, la incidencia de pacientes que necesitó terapias de remplazo renal había aumentado considerablemente, influenciado notablemente por el incremento paralelo de dos patologías en todo el mundo: la HTA y la DM (2).

La diálisis consiste en un método depurativo de toxinas, electrolitos y exceso de agua del organismo, que se puede ejecutar a través de dos técnicas, una de ellas corresponde a la hemodiálisis (HD) que implica el uso de un filtro (membrana artificial) a través del cual pasa la sangre y es retornada al paciente limpia, mientras que la diálisis peritoneal (DP) usa como filtro depurativo una membrana natural que corresponde a la membrana peritoneal que reviste la pared y los órganos abdominales, no usa sangre, sino que líquido dializante con un agente osmótico que atrae toxinas y agua para finalmente eliminarlas (3), ambas modalidades presentan una inci- dencia y prevalencia crecientes en las últimas décadas.

La única manera de recuperar la función renal es mediante el trasplante renal (TR), sin embargo no siempre es posible que un paciente sea susceptible de trasplantarse (4), por tanto el paciente debe escoger necesariamente entre DP y HD.

Hoy en día constituye un derecho de los pacientes recibir información de los tratamientos que existen para que puedan participar con el equipo terapéutico en la elección de la técnica dialítica (5); para ello los pacientes deben tener conocimientos relativos a las ventajas y desventajas de cada modalidad terapéutica, y poder conjugarlas con los intereses personales. La diálisis peritoneal en Chile, como técnica de tratamiento sustitutivo desde la década del 60 , ha evolucionado con el paso de los años, siendo necesario actualizar los nuevos aspectos beneficiosos para el paciente, que pueden hacer de ella un instrumento terapéutico de primer orden (6).

En Chile, el tratamiento sustitutivo de la enfermedad renal crónica (ERC) en etapa 5 se encuentra en un $72,4 \%$ cubierto por el Sistema de Salud Pública, incluida en las patologías con Garantías Explícitas de Salud (GES) (7).

Reportes de la Sociedad Chilena de Nefrología indican que en nuestro país la prevalencia de pacientes en el programa de DP ha ido en aumento (60 pacientes al 31 de diciembre de 2008, ampliándose a 907 al 31 de diciem- 
bre de 2012) (8), a pesar de ser considerablemente menor que la existente en hemodiálisis (13.625 al 31 de agosto de 2008 (9), elevándose a 17.014 personas al 31 de agosto de 2012) (10), este crecimiento en diálisis peritoneal se encuentra asociado a la incorporación de la técnica dialítica al GES.

La enfermedad renal altera todo el equilibrio de la persona y su medio, sometiéndola a diversos factores estresantes, provocándole diversos grados de dependencia, en el ámbito físico, sicológico y social, por tanto es preciso conocer las necesidades de esta población para dar cobertura a sus requerimientos y favorecer en los equipo de salud tratantes un mejor abordaje, basado en el enfoque clínico, la persona y su entorno, desde una perspectiva integral.

La función propia de la enfermería es asistir al individuo, sano o enfermo, en la realización de aquellas actividades que contribuyen a la salud o su recuperación (o a la muerte pacífica), actividades que la persona realizaría si tuviera la fuerza, voluntad o el conocimiento necesario, y hacerlo de tal manera que le ayude a ganar independencia a la mayor brevedad posible. "El foco de actuación de la enfermera es el área de dependencia de la persona"(11).

Henderson $(11,12)$ refiriéndose a la práctica de la profesión, indica que la enfermera, cuya función principal consiste en ser la cuidadora directa del paciente, se verá recompensada a medida que el paciente vaya ganando independencia; debe esforzarse por comprenderlo, cuando éste carece de voluntad, conocimiento o fuerza. Tal y como Henderson afirmó debe "ponerse en su piel". Normalmente las necesidades se encuentran satisfechas por la persona, cuando tiene: la fuerza, el conocimiento y la voluntad para cubrirlas (independiente), pero cuando algo de esto falta o falla en la persona una o más necesidades no se satisfacen, surgiendo los problemas de salud (dependiente: fuentes de dificultad), es entonces cuando la enfermera tiene que ayudar o asistir a la persona para que pueda satisfacerlas.

Por lo citado anteriormente, el profesional de enfermería puede ayudar al paciente a avanzar hacia la independencia, valorando, diagnosticando, realizando intervenciones y evaluando cada uno de los 14 elementos del cuidado. De ahí la importancia que este modelo sea considerado en este estudio, ya que la diálisis peritoneal posee un claro enfoque en que el paciente en su condición de enfermedad busque la autonomía y la forma de recuperar un estado óptimo de salud.

El siguiente estudio pretende conocer las características biosociodemográficos y la satisfacción de necesidades de los pacientes en tratamiento con diálisis peritoneal del Hospital Las Higueras de Talcahuano, Chile.

\section{MATERIAL Y MÉTODO}

Estudio, cuantitativo, descriptivo, de corte transversal, consistente en la aplicación de un instrumento a través de una encuesta en el domicilio del paciente, el cual constó de dos ítemes, uno relacionado con los aspectos biosociodemográficos y el otro con la Valoración de Necesidades Básicas de Virginia Henderson, quedando configurado en un cuestionario con un total de 25 preguntas con respuestas cerradas formato likert. El universo estuvo constituido por 42 pacientes que se atendían en la Subunidad de DP del Centro de Costo (CC) de Diálisis y Trasplante Renal del Hospital Las Higueras de Talcahuano al 31 de marzo de 2012, excluyéndose a usuarios que no presentaban adherencia al tratamiento (interrupción de la terapia automática y /o manual por parte del paciente y constatado por condición clínica y exámenes de laboratorio) y a quienes no desearon participar. De este modo, la población del estudio quedó conformada por 40 pacientes, quienes previo consentimiento informado accedieron a ser parte de este estudio. El proyecto fue sometido a revisión del Comité de 
Ética de la Facultad de Medicina de la Universidad de Concepción y al jefe del Centro de Costo de Diálisis y Trasplante Renal del Hospital Las Higueras de Talcahuano.

Los datos obtenidos fueron codificados, tabulados y presentados en una base de datos del software Microsoft Office Excel, versión 2010. El análisis de los datos se llevó a cabo a través de estadística descriptiva y medidas de tendencia central.

\section{RESULTADOS}

El perfil de la muestra estuvo constituida en partes iguales por hombres y mujeres, el promedio de edad correspondió a 48 años, el $10 \%$ tiene más de 65 años, más de la mitad vive sin pareja $(55,0 \%)$ y es jefe de hogar $(57,5 \%)$, la mayoría tiene hijos $(75,0 \%)$, predominando los grupos familiares integrados por 4 o más personas $(57,5 \%)$, la totalidad declara tener creencia en un ser superior y pertenecer a una religión. El mayor porcenta- je de ellos ha cursado la enseñanza media, un $22,5 \%$ se encuentra trabajando y recibe una remuneración, el resto principalmente percibe ingresos por concepto de pensión y ayuda de familiares, declarando el 50\% de ellos que la satisfacción económica es medianamente insuficiente, el $31,7 \%$ es analfabeto y el $45 \%$ es alfabeto, y solo el $23,3 \%$ ha recibido educación formal. La gran mayoría cuenta con saneamiento básico en sus domicilios y vive en sector urbano.

En relación a la satisfacción de necesidades formulada por Virginia Henderson, respirar con normalidad, comer y beber, moverse, dormir y reposar vestirse y desvestirse, mantención de la temperatura corporal, cuidar su presencia, comunicación, actuar según sus creencias y valores, trabajar para realizarse, recrearse y aprender y considerando los indicadores de dependencia e independencia, reportaron valores entre el $57,5 \%$ y $92,5 \%$ de satisfacción; entre éstas las que presentaron menor grado de satisfacción fueron mantener la temperatura corporal y recrearse, ambas con $57,5 \%$ (Gráfico 1).

Gráfico 1. Distribución de Valoración de Necesidades según Virginia Henderson en pacientes que realizan diálisis peritoneal, Hospital Las Higueras 2012, Talcahuano.

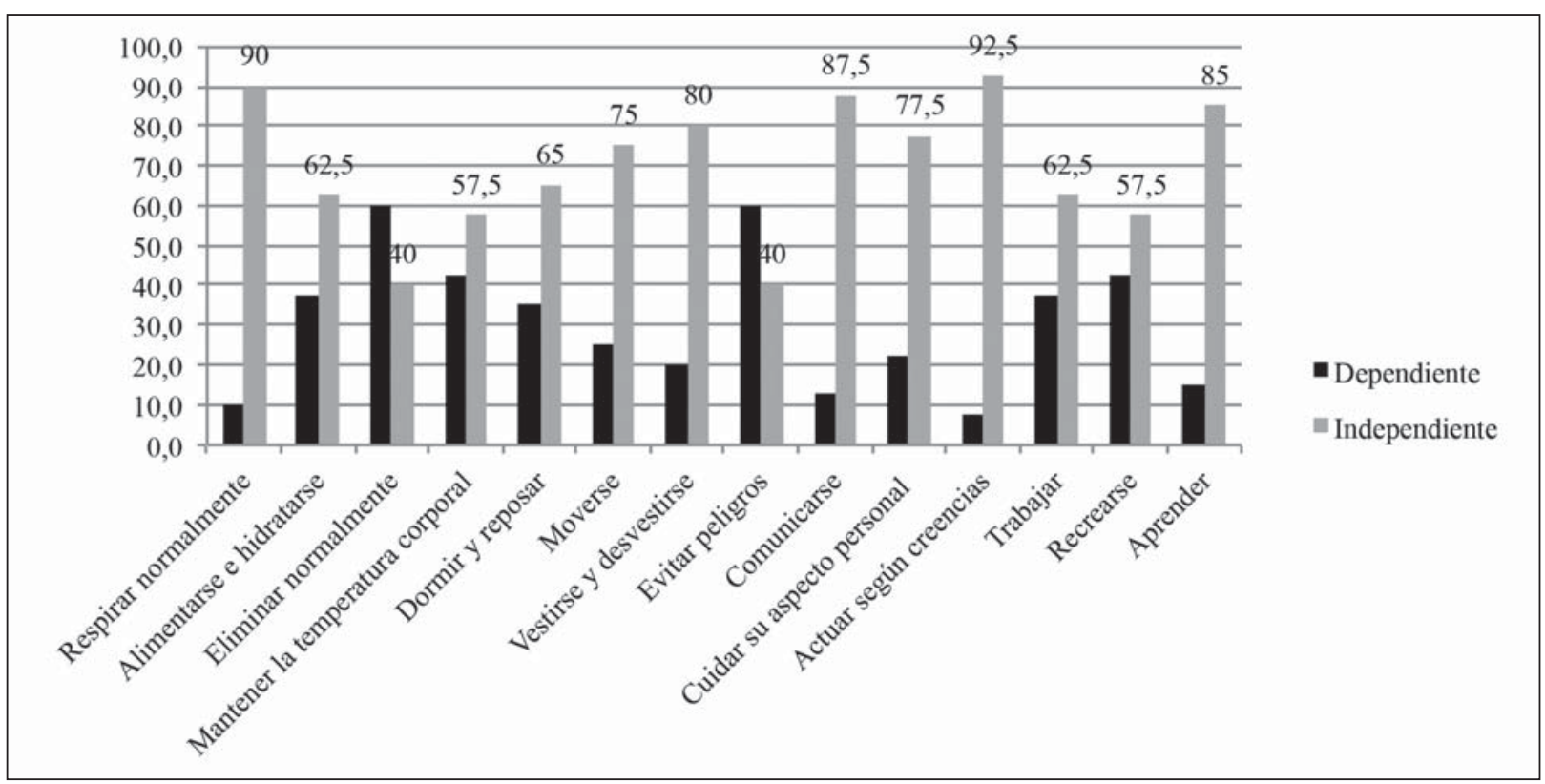




\section{DISCUSIÓN Y CONCLUSIÓN}

Considerando el sexo, nuestros resultados se asemejan a Duarte et al. (13), encontrando que el 50,1\% de los pacientes en diálisis peritoneal fueron hombres, pero el doble de su población correspondieron a mayores de 65 años $(28,2 \%)$, similar a lo publicado por Carrillo y Díaz (14) en su estudio piloto en Bogotá, cuyo objeto fue conocer la capacidad de autocuidado de los pacientes y su relación con las características sociodemográficas, siendo el 51,7\% de los participantes del estudio hombres.

Remón et al. (15) presentaron las características de la población incluida en diálisis peritoneal durante 10 años, siendo el 57\% de ellos hombres, promedio de edad 58 años, lo que es superior a lo descrito en este artículo; no mencionan antecedentes sobre ruralidad, ni nivel educacional. Varela (16) mostró similares características para sexo y edad, pero agregó que el 47,2\% de los estudiados tenía residencia en zona rural, lo que difiere de los hallazgos de este estudio, donde la ruralidad correspondió al 10\%; en lo que respecta a la situación de pareja, a diferencia de nuestros resultados, el 64,2\% se encuentra en pareja, finalmente en cuanto a la actividad remunerada, el $32,7 \%$ no se encuentra trabajando, similar a los resultados de nuestro estudio. Semejantes resultados presentó Carrillo y Díaz (14), encontrando que el 68,7\% de los pacientes estaba en pareja, sin embargo, difiere en cuanto a la actividad laboral, ya que el 51,72\% de los pacientes de su estudio se encontraba trabajando.

En relación a la valoración de necesidades, se observó que "eliminar bien" y "evitar los peligros" se encuentran insatisfechas en un $60 \%$, mientras que las 12 necesidades restantes se encuentran satisfechas en al menos el 57,5\% de los entrevistados, entre estas últimas, las que presentan un menor grado de independencia, corresponden a "mantener la temperatura corporal" y "recrearse", similar a lo identificado por Puebla(17) en 62 pacientes de hemodiálisis de Valparaíso, donde señaló que el 58,1\% de los pacientes de hemodiálisis que fueron parte de su estudio refirieron sentirse muy limitados para realizar esfuerzos intensos, sin embargo la mayoría (65\%) declaró que podían realizar actividades de la vida diaria sin mayores problemas y sin que el dolor percibido afectara esto. Semejante es lo presentado por Carrillo y Díaz (14), donde el 57\% de los estudiados declaró "hacer ejercicio", agregando además que "percibían un buen estado de salud", de los restantes, 46 personas señalaron que casi siempre piensan en hacer ejercicio, pero no logran llegar a hacerlo; desde otra perspectiva es necesario destacar que entre los pacientes que presentaron deficiente capacidad de autocuidado se encuentran quienes se desempeñan en oficios varios o como empleados, que se encuentran solos o sin pareja, lo que, según los autores, podría asociarse a una falta de red de apoyo que los inste a cuidarse. Se opone a estos hallazgos lo señalado por Seguí et al. (18), quienes evaluaron la calidad de vida en un grupo de pacientes en hemodiálisis de Tarragona, en donde la condición basal de salud junto con ser mayor de 65 años y encontrarse viudo o separado llevaron a alterar las 8 dimensiones evaluadas, lo que indirectamente puede señalar una baja satisfacción de necesidades, que por sí mismos no pueden cubrirlas, probablemente tampoco lo harán con ayuda externa, producto de relacionarse menos con otras personas.

La mantención de la temperatura en este paciente se ve alterada por múltiples factores: deficiencias en la vascularización, alteración en la distribución de los líquidos corporales y perfusión tisular disminuida, entre otros, lo que conlleva a un estado de hipotermia leve permanente con temperaturas promedio de $35,5^{\circ} \mathrm{C}$. La recreación se ve afectada por la dependencia al tratamiento, la ausencia de amigos al no poder adaptarse a ellos, el deterioro de las relaciones de pareja o la imposibilidad de generarlas se convierten en las ansiedades 
que marcan su vida (19).

Eliminar normalmente obtuvo una puntuación de un $40 \%$, lo revierte un gran dilema, ya que por un lado este paciente está sometido a restricciones permanentes en la ingesta de líquidos y alimentos para mantener un óptimo control hemodinámico y por otro lado inevitablemente evoluciona hacia la anuria y estreñimiento intermitente, como consecuencia de la enfermedad base, esto concuerda con lo presentado por Gil Gil et al. (20), quienes a partir de la información aportada por 40 personas que recibían hemodiálisis, de distintas nacionalidades, concluyeron que las restricciones dietéticas y limitaciones de actividad física se comportaron como un factor de riesgo para el desarrollo de estreñimiento, destacando que el uso de quelantes de fósforo, antiácidos y suplementos de hierro favorecieron su aparición, ya que es un efecto inherente al uso de éstos, lo anterior toma mayor importancia si se considera lo planteado por Carrillo y Díaz (14) que identificaron que el $62 \%$ de los pacientes tiene buen compromiso con el tratamiento farmacológico y un $78 \%$ de los estudiados cumple la dieta alimenticia que se les asigna.

Evitar los peligros presentó un 40\% de satisfacción, lo que es esperable, dado que el usuario en diálisis debe aprender a manejar recursos que no conoce, lo que implica tener que vivir y asumir una serie de restricciones $y$ dificultades de forma continua, relacionadas con: la enfermedad, estado físico, características de la diálisis y estado de cronicidad, lo que afecta sus emociones, estados de ánimo y sentimientos de dependencia.

Finalmente, la evaluación de las necesidades que presentan mayor independencia y por consiguiente se encuentran satisfechas generan una importante contribución, ya que durante la revisión bibliográfica no se encontró literatura al respecto.

Actualmente, en el ámbito de la enfermería en el país no se ha estudiado la Valoración de necesidades en pacientes con terapia dialítica, utilizando un Modelo de Enfermería, lo que suponemos se debe a que recién la Diálisis Peritoneal se está iniciando como terapia de elección de sustitución renal (GES), por tanto el profesional de enfermería que trabaja en los cuidados directos e integrales del usuario en esta terapia debe dirigir su quehacer hacia la satisfacción de las necesidades, para favorecer las condiciones de vida de estos pacientes y su entorno familiar más cercano.

Concluimos que los resultados de este estudio sugieren: implementar y reforzar planes educativos más rigurosos a los ya existentes en nuestro centro asistencial, tendientes a evitar daños físicos y sicológicos, así como también mantener una buena eliminación corporal, a través de la retroalimentación en cada control o visita domiciliaria realizada al usuario, familia y redes de apoyo, y continuar investigando el tema permanentemente, cuya finalidad es ir conociendo la problemática que enfrentan los pacientes y su familia sometidos a esta terapia dialítica en nuestro medio y a partir de esto otorgar un cuidado de calidad humanizado, lo que se reflejará en una mejora en la calidad de vida del paciente, familia y comunidad.

\section{REFERENCIAS}

1. Valderrabano F. Tratado de hemodiálisis. Barcelona: Editorial médica. JIMS.SL 1999-568 p.

2. Sociedad española de nefrología, Sociedad española de enfermería nefrológica. Manual Práctico de Diálisis Peritoneal. España. 2003. 27-92.

3. Daugirdas J. Manual de Diálisis. 2da ed. Barcelona: Masson S.A; 2001. 15, 231 p.

4. Montenegro J, Correa-Rotter R, Riella M. Tratado de Diálisis Peritoneal. Barcelona: Elsevier; 2009. 93-95 p.

5. Sandi M, Romay N, Ríos C, Kuszmiruk A. Diálisis Peritoneal: complicaciones más frecuentes. Rev. posgrado VIa Cátedra 
Med. 2010; (3): 16-21.

6. García M, Rodríguez A, Ponz E, Almirall J. Opciones ante la insuficiencia renal crónica terminal en un hospital comunitario. Nefrología 1995; 15; 349-355.

7. Ministerio de Salud. Guía Clínica Diálisis Peritoneal. Santiago, Chile. 2010. 9-88.

8. Poblete H. Sociedad Chilena de Nefrología Registro de diálisis. XXVIII Cuenta de Hemodiálisis Crónica (HDC) en Chile [Internet]; 2008. [citado 1 noviembre 2012]. Disponible en: http://www.asodi. $\mathrm{cl}$

9. Poblete H. Sociedad Chilena de Nefrología Registro de diálisis. XXXI Cuenta de Hemodiálisis Crónica (HDC) en Chile [Internet]; 2012. [citado 1 noviembre 2012]. Disponible en: http://www.asodi.cl

10. Valenzuela M, Ortiz M. Sociedad Chilena de Nefrología. Informe de Diálisis Peritoneal en Chile [Internet]; 2013. [citado 15 de octubre 2013]. Disponible en: www. asodi.cl

11. Henderson V. The nature of nursing. Am J Nurs. 1964; 64 (8): 62-8.

12. Marriner A, Raile M. Modelos y Teorías de Enfermería. $5^{\circ}$ Edición. Barcelona: Elsevier Mosby; 2007. 98-107 p.

13. Duarte J, Ribeiro K, Andrade M, Andrade K. Preditores de peritonite em pacientes em um programa de diálise peritoneal. J Bras Nefrol. 2010. 32(2): 156-164.

14. Carrillo A, Díaz J. Capacidad de autocuidado de los pacientes en diálisis perito- neal: un estudio piloto en Bogotá. Enfermería Global. 2013. 12(2): 54-64.

15. Remón C, Quirós PL, Gil JM, Ros S, Aresté N, Ruiz A, et al. Diez años de diálisis peritoneal en Andalucía (1999-2008): datos epidemiológicos, tipos de tratamiento, peritonitis, comorbilidad y supervivencia de pacientes y técnica. Nefrología 2010; 30(1): 46-53.

16. Varela L, Vázquez M, Bolaños L, Alonso R. Predictores psicológicos de la calidad de vida relacionada con la salud en pacientes en tratamiento de diálisis peritoneal. Nefrología 2011: 31(1): 97-106.

17. Puebla P. Calidad de vida y características personales de los pacientes insuficientes renales crónicos terminales en tratamiento de hemodiálisis en centros públicos y privados de la $\mathrm{V}$ Región. Revista de la Sociedad Chilena de Enfermería y Transplante Renal 2011: 6(11): 7-14.

18. Seguí A, Amador P, Ramos A. Calidad de vida en pacientes con insuficiencia renal crónica en tratamiento con diálisis. Rev Soc Esp Enfer Nefrol. 2010: 13(3). 155160.

19. Andreau Periz L, Force San Martin E. 500 cuestiones que plantea el cuidado del enfermero renal. 2da edición. Barcelona: Masson; 2005. 373 p.

20. Gil Gil A, Gumbao A, Canales A. Incidencia de estreñimiento en una unidad de hemodiálisis. Rev Soc Esp Enfer Nefrol. 2006: 9(3): 172-176. 